\title{
Sex-specific differences in endoplasmic reticulum aminopeptidase 1 modulation influence blood pressure and renin- angiotensin system responses
}

Sanjay Ranjit, Jian Yao Wong, Jia W. Tan, Chee Sin Tay, Jessica M. Lee, Kelly Yin Han Wong, Luminita H. Pojoga, Danielle L. Brooks, Amanda E. Garza, Stephen A. Maris, Isis Akemi Katayama, Jonathan S. Williams, Alicia Rivera, Gail K. Adler, Gordon H. Williams, and Jose R. Romero

Division of Endocrinology, Diabetes and Hypertension, Department of Medicine, Brigham and Women's Hospital and Harvard Medical School, Boston, Massachusetts, USA.

Salt sensitivity of blood pressure (SSBP) and hypertension are common, but the underlying mechanisms remain unclear. Endoplasmic reticulum aminopeptidase 1 (ERAP1) degrades angiotensin II (ANCII). We hypothesized that decreasing ERAP1 increases BP via ANGII-mediated effects on aldosterone (ALDO) production and/or renovascular function. Compared with WT littermate mice, ERAP1-deficient (ERAP1 ${ }^{+-}$) mice had increased tissue ANGII, systolic and diastolic BP, and SSBP, indicating that ERAP1 deficiency leads to volume expansion. However, the mechanisms underlying the volume expansion differed according to sex. Male ERAP1 ${ }^{+-}$mice had increased ALDO levels and normal renovascular responses to volume expansion (decreased resistive and pulsatility indices and increased glomerular volume). In contrast, female ERAP1+/- mice had normal ALDO levels but lacked normal renovascular responses. In humans, ERAP1 rs30187, a lossof-function gene variant that reduces ANGII degradation in vitro, is associated with hypertension. In our cohort from the Hypertensive Pathotype (HyperPATH) Consortium, there was a significant dose-response association between rs30187 risk alleles and systolic and diastolic BP as well as renal plasma flow in men, but not in women. Thus, lowering ERAP1 led to volume expansion and increased BP. In males, the volume expansion was due to elevated ALDO with normal renovascular function, whereas in females the volume expansion was due to impaired renovascular function with normal ALDO levels.

Conflict of interest: The authors have declared that no conflict of interest exists.

Copyright: (c) 2019, American Society for Clinical Investigation.

Submitted: April 24, 2019 Accepted: September 19, 2019 Published: November 1, 2019.

Reference information: /CI Insight. 2019;4(21):e129615.

https://doi.org/10.1172/jci. insight.129615.

\section{Introduction}

Among the major issues that need to be addressed in the treatment of hypertension (HTN) are the unclear mechanisms by which environmental factors, particularly sodium intake, affect BP. Growing evidence suggests that SSBP is a substantial risk factor for cardiovascular (CV) morbidity and mortality $(1,2)$. Recent studies underscore the need to precisely define the mechanisms by which sodium intake influences CV function $(3,4)$. A potential mechanism centers around endoplasmic reticulum aminopeptidase 1 (ERAP1), which metabolizes angiotensin II (ANGII) and, thus may influence BP homeostasis though mechanisms that remain unclear $(5,6)$.

ERAP1 is a zinc metallopeptidase also known as adipocyte-derived leucine aminopeptidase (ALAP), aminopeptidase regulator of TNFR1 shedding 1 (ARTS-1), endoplasmic reticulum aminopeptidase associated with antigen processing (ERAAP), and puromycin-insensitive leucyl-specific aminopeptidase (PILSAP) $(7,8)$. It is a multifunctional enzyme widely expressed in the body. Its role in the immune system and the pathophysiology of ankylosing spondylitis has been most extensively studied, since it can cleave cytokine cell surface receptors and trim HLA class-binding precursors (9-11).

ERAP1 rs30187, a loss-of-function human genetic variant, is associated with reduced degradation of ANGII in vitro (5-7) and with essential HTN in a cohort of Japanese study participants (12). More recent data suggest that elevated ERAP1 mediates the hypotensive response to sepsis by increasing metabolism of ANGII (13). These effects on ANGII led us to reason that a loss-of-function mutation of the ERAP1 gene would lead to increased BP by affecting ANGII-responsive processes - aldosterone (ALDO) secretion and/or 
modulation of renovascular function. We used 2 approaches to test this hypothesis. First, we assessed measures of BP homeostasis, renin-angiotensin system (RAS) activity, and renovascular function in a mouse deficient in 1 ERAP1 allele (ERAP1 ${ }^{+/}$) - a model with expression likely to be similar to what occurs in humans, i.e., reduced, but not absent, ERAP1. Second, we performed a gene association study on the previously reported single nucleotide variant rs30187 of ERAP1 in a cohort of carefully phenotyped individuals participating in the Hypertensive Pathotype (HyperPATH) Consortium (14-16). Finally, we assessed whether biological sex modifies the responses observed.

\section{Results}

\section{Mouse studies}

ERAP1 expression levels are reduced in ERAP1 $1^{+/-}$mice. We developed a colony of ERAP1 ${ }^{+/-}$and WT littermate control mice. We studied female and male mice between 18 and 21 weeks of age (Supplemental Table 1; supplemental material available online with this article; https://doi.org/10.1172/jci.insight.129615DS1). We used reverse transcriptase PCR (RT-PCR) to measure ERAP1 mRNA levels in renal cortices and heart tissues isolated from ERAP1 $1^{+/-}$mice. Compared with WT mice, ERAP1 $1^{+/-}$mice had approximately $50 \%$ lower ERAP1 mRNA levels in both tissues (Figure 1, A and B). Consistent with these data, aorta mRNA levels were reduced by about $50 \%$ in $\mathrm{ERAP}^{+/-}$as compared with WT mice (Supplemental Figure 1). In addition, Western blot analyses showed that ERAP1 protein levels in heart and kidney were likewise reduced by approximately $50 \%$ in ERAP $1^{+/-}$mice when compared with WT littermates (Figure 1, C and D). A similar reduction in ERAP1 was observed in spleen (Supplemental Figure 2).

Increased tissue ANGII levels in ERAP1+/- mice. ERAP1 has been shown to degrade ANGII in vitro (5-7). Thus, lowering ERAP1 would be expected to increase tissue ANGII levels. We used liquid chromatography-mass spectroscopy (LC-MS) to measure ANGII levels ex vivo in aorta, kidney, and heart tissues of WT and ERAP1 ${ }^{+/-}$mice on a liberal-salt diet. Our results show that compared with WT mice, ERAP1 ${ }^{+/-}$mice had approximately twice the levels of tissue ANGII (WT: $29.6 \pm 6.7 \mathrm{fg} / \mathrm{mg}$ of tissue, $n=7$; ERAP1 $1^{+/-}: 58.1$ $\pm 19.7 \mathrm{fg} / \mathrm{mg}$ of tissue, $n=9$; mean \pm SEM, $P=0.029)$. The difference between WT and ERAP $1^{+/-}$varied by tissue, with ANGII levels in ERAP1 ${ }^{+/}$mice being $44 \%$ higher in the heart, $68 \%$ higher in the kidney, and approximately 6 times higher in the aorta.

$E R A P 1^{+/-}$mice have increased BP and SSBP. We measured BP by tail-cuff plethysmography in WT and ERAP $1^{+/-}$mice after 7 days of either a liberal-salt diet $(1.6 \%$ sodium $)$ or a restricted-salt diet $(0.03 \%$ sodium). We show that compared with WT mice, ERAP $1^{+/-}$mice had higher systolic BP and diastolic BP on a liberal-salt diet, and these and these were similar between female and male mice (Figure 2, A-D). However, when mice were placed on a restricted-salt diet, there was a significant reduction, with similar BP levels in both genotypes and sexes (Figure 2). We estimated salt sensitivity of BP (SSBP) in these mice ( $\Delta$ systolic $\mathrm{BP}=$ systolic $\mathrm{BP}$ on $1.6 \%$ sodium diet minus systolic $\mathrm{BP}$ on $0.03 \%$ sodium diet). ERAP $1^{+/-}$mice had significantly greater SSBP than WT littermates (Figure 2, E and F). We then measured BP by radiotelemetry from transducers implanted in the carotid artery of unrestrained mice. As observed by tail-cuff, SSBP was greater in ERAP1 ${ }^{+/-}$compared with WT mice (Supplemental Figure 3).

Effects of ERAP1 deficiency on urinary hormone and electrolyte levels. In response to ANGII, the adrenal gland produces and releases ALDO. We measured 24-hour urinary ALDO levels. ALDO levels were increased in male but not female ERAP $1^{+/-}$compared with WT mice after 1 week of a $1.6 \%$ sodium diet (Figure 3, A and B). As expected, lowering sodium intake to $0.03 \%$ for 1 week caused a significant increase in urinary ALDO levels in mice of both genotypes that was greater in female than male mice (Figure 3, C and D). Measures of plasma renin activity (PRA) showed that male levels were greater in ERAP $1^{+/-}$mice than WT littermates $(85.4 \pm 9.0$ vs. $67.9 \pm 8.4 \mathrm{ng} / \mathrm{mL} / \mathrm{h}$, mean $\pm \mathrm{SEM}, P=0.047)$, a difference that was also observed in female ERAP1 ${ }^{+/-}$compared with WT mice $(81.7 \pm 9.0 \mathrm{vs} .67 .4 \pm 22.7 \mathrm{ng} / \mathrm{mL} / \mathrm{h}$, mean \pm SEM, $P=0.05$ ). We did not observe any significant differences in urinary sodium, potassium, albumin, or creatinine levels between WT and ERAP1 ${ }^{+/-}$mice (data not shown).

ERAP1 deficiency affects renovascular function. Another proximate mechanism that may lead to increased $\mathrm{BP}$ in $\mathrm{ERAP}^{+/-}$mice is altered renovascular activity (17). To determine the effects of ERAP1 deficiency on measures of renovascular function, we used Doppler ultrasound of left renal arteries in anesthetized mice to measure resistive index (RI) and pulsatility index (PI). These indices are calculated from renal artery flow velocities during cardiac systole and diastole and have been used to estimate renovascular resistance, 

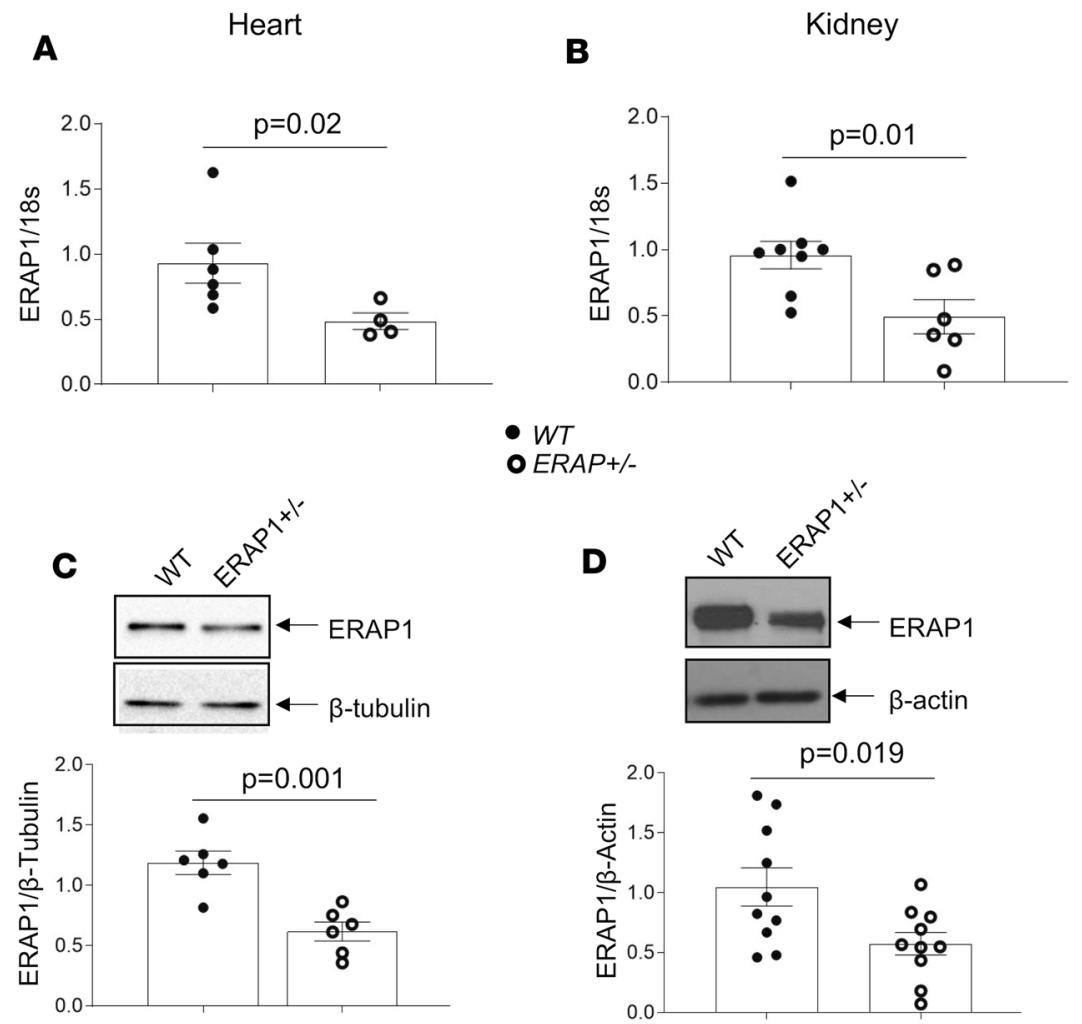

Figure 1. ERAP1 levels are reduced in ERAP1 ${ }^{+/-}$compared with WT littermate control mice. (A) ERAP1 mRNA levels in heart tissue by RT-PCR (WT $n=6$, ERAP1 ${ }^{+-} n=4 ; P=0.02$ ). (B) ERAP1 mRNA levels in renal cortex by RT-PCR (WT $n=8$, ERAP1 ${ }^{+/-} n=6 ; P=0.01$ ). (C) Western blots and representative optical densitometry of ERAP1 in heart tissue, normalized to $\beta$-tubulin (WT $n=6$, $\operatorname{RAP}^{+/-} n=6 ; P=0.001$ ). (D) Western blot and representative optical densitometry of ERAP1 in kidney tissue, normalized to $\beta$-actin (WT $n=10, E R A P^{+/-} n=10 ; P=0.019$ ). There were no differences by sex. Data are presented as mean \pm SEM; 2-tailed Student's $t$ test.

and they are inversely correlated with measures of renal plasma flow (RPF) (18). Our results in ERAP1 ${ }^{+/}$ mice show that males, but not females, had lower RI and PI compared with WT mice (Figure 4) - measures that suggest increased RPF in male but not female ERAP1 ${ }^{+/}$mice. Increased RPF is the anticipated physiologic response to an increase in vascular volume. We also estimated glomerular volume as another marker of renal response to a liberal-salt diet. Again, in ERAP1 ${ }^{+/-}$compared with WT mice, there was a significant difference by sex: males had significantly larger glomeruli, while females had significantly smaller glomeruli than WT littermates (Figure 5). In addition, male ERAP1 ${ }^{+/}$mice had increased kidney mass/body mass and heart mass/body mass ratios compared with male WT mice (Figure 6). No significant differences in these ratios were noted between female ERAP $1^{+/-}$and female WT mice. However, male ERAP $1^{+/-}$mice had body mass similar to that of male WT mice, and both were larger than female mice of either genotype (Figure 6).

\section{Human studies}

Human subject characteristics. In our human studies, we controlled several environmental variables that could confound the interpretation of our results. Our studies were performed under standardized conditions of time of day, liberal-salt diet, withdrawal of interfering BP medications, and overnight supine posture. Our analyses were performed with participation of individuals from the HyperPATH cohort (a cohort used frequently to assess deep phenotype-genotype associations in HTN) who were genotyped for the at-risk variant rs30187 of ERAP1 and for whom we had complete phenotype data. Following maintenance of supine posture and fasting overnight, BP, PRA and ALDO, and urine ALDO, sodium, and potassium values were obtained between 8 am and $10 \mathrm{am}$. Demographic characteristics are shown in Table 1 . There were no significant differences in ALDO, age, BP, BMI, and sex between the allelic groups at rs 30187 in this cohort. 
Female
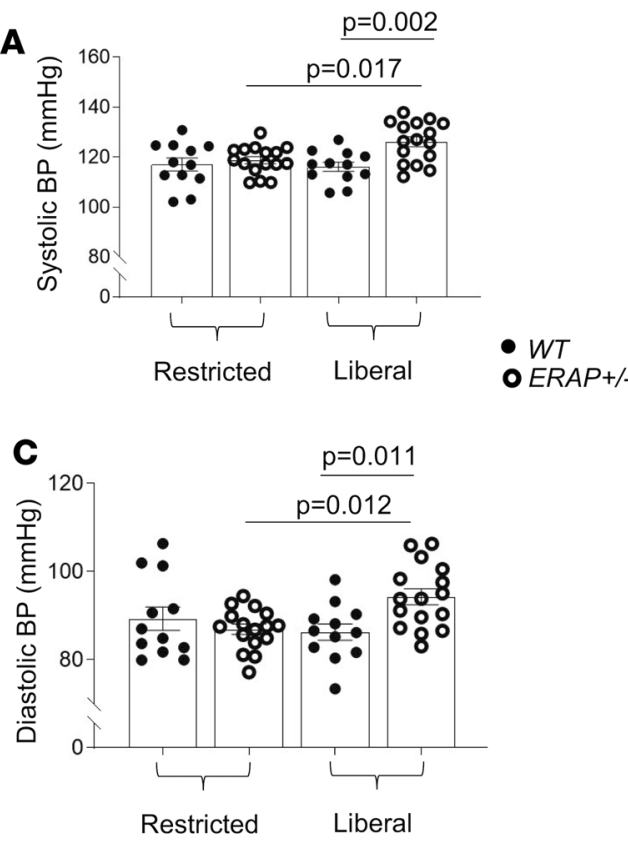

$\mathbf{E}$

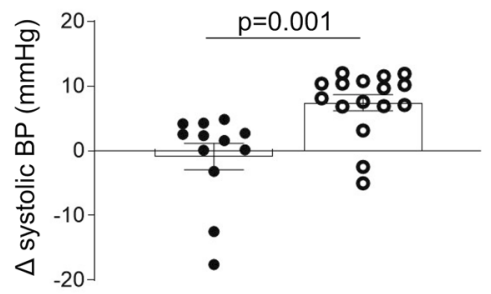

Male

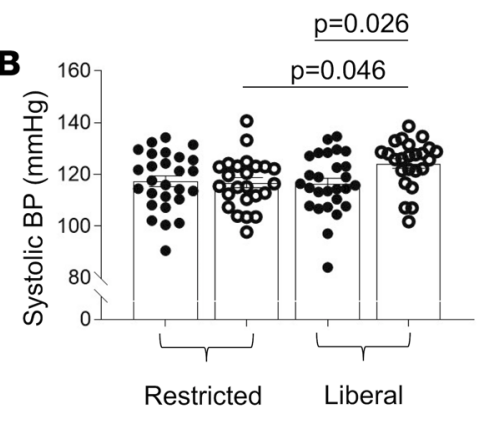

D

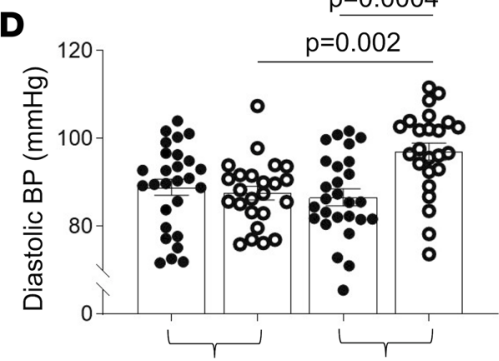

Restricted Liberal

$\mathbf{F}$

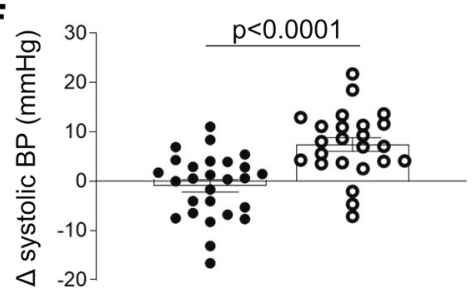

Figure 2. Effect of 1 week of restricted-salt diet and 1 week of liberal-salt diet on systolic and diastolic BP in female and male ERAP1 ${ }^{+/-}$and WT mice. Systolic ( $\mathbf{A}$ and $\mathbf{B}$ ) and diastolic ( $\mathbf{C}$ and D) BP by tail-cuff measurements after 1 week on a restricted-salt diet and liberal-salt diet (Liberal). Measurements of SSBP (E and $\mathbf{F}$ ) ( $\triangle$ systolic $\mathrm{BP}=$ systolic $\mathrm{BP}$ on liberal minus systolic $\mathrm{BP}$ on restricted-salt diet). Filled circles indicate WT mice; open circles, ERAP1 ${ }^{+/-}$mice. Data are presented as mean $\pm \mathrm{SEM}$. WT $n=12-27$, ERAP1 ${ }^{+/-} n$ = 16-24; 1-way ANOVA for multiple groups (A-D) and 2-way Student's $t$ test for paired comparisons ( $E$ and $\mathbf{F}$ ).

Association of ERAP1 rs30187 with BP and RPF in human subjects on a liberal-salt diet. We assessed the relationship of genotype to systolic and diastolic BP and RPF by the para-aminohippurate (PAH) clearance method. There was a significant allele dose response in systolic and diastolic BP on a liberal-salt diet (200 $\mathrm{mmol} / \mathrm{d}$ ) in men that was not observed in women (Figure 7). Systolic BP in male risk allele homozygotes was approximately $20 \mathrm{mmHg}$ higher than in female risk allele homozygotes and $14 \mathrm{mmHg}$ greater than in male non-risk allele carriers (homozygous for the major allele of rs30187).

Consistent with the results in male ERAP $1^{+/-}$mice, a significant positive allele dose response in RPF on a liberal-salt diet (200 mmol/d) was observed in men but not women (Figure 8). Risk allele-homozygous men had RPF that was approximately $50 \mathrm{~mL} / \mathrm{min} / 1.73 \mathrm{~m}^{2}$ higher than that of risk allele-homozygous women or male non-risk allele carriers.

Expression of ERAP1 in human CV tissue. We employed an in silico approach to examine rank normalized gene expression levels in humans of ERAP1 rs30187 in adrenal gland, aorta, tibial artery, and coronary artery using the Genotype-Tissue Expression (GTEx) Project database for analyses of expression quantitative trait loci (eQTLs) (19). For all tissues analyzed, mRNA expression related to the number of risk alleles that were highly significantly expressed (between $P=0.00032$ and $P=8 \times 10^{-12}$ ), with effect sizes of -0.32 to -0.47 (Supplemental Table 2). Thus, the greater the number of alleles, the lower the level of ERAP1 mRNA, suggesting that rs30187 was associated with reduced ERAP1 levels in CV and adrenal tissues.

\section{Discussion}

Our results support the hypothesis that ERAP1 deficiency leads to increased ANGII levels and increased BP. In this study, using genetically engineered mice, we characterized 2 likely key proximate BP homeostatic mechanisms that modulate sodium reabsorption and volume expansion: (i) ALDO secretion (urinary ALDO levels); and (ii) renovascular function (RI and PI of the left renal artery). Dysfunction in one or both 

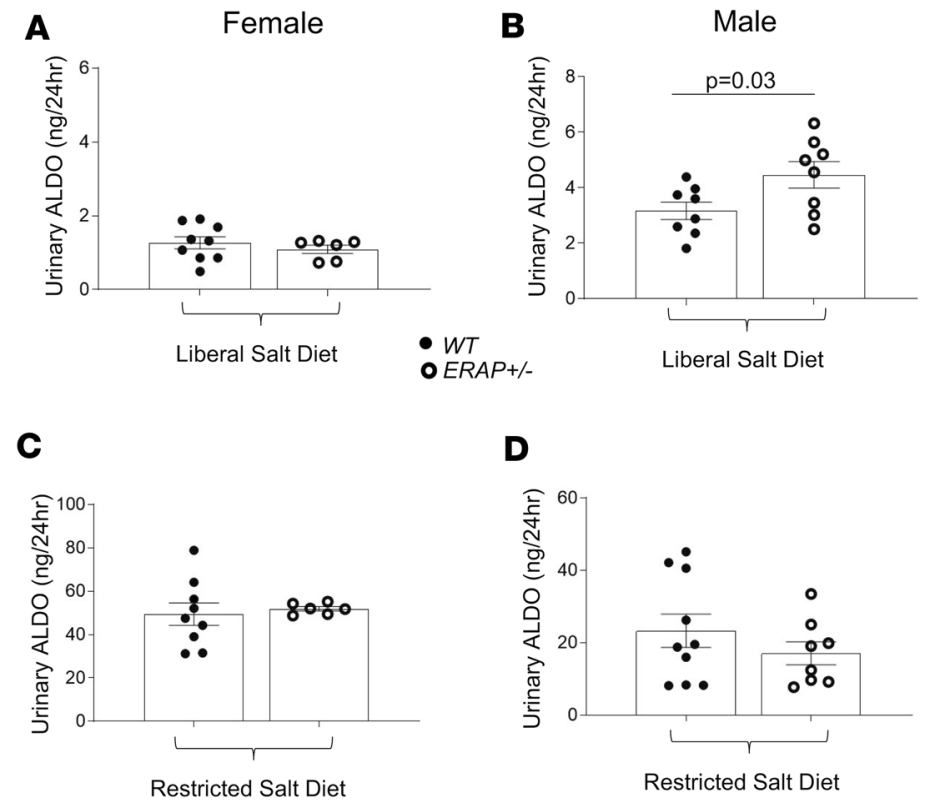

Figure 3. Urinary ALDO levels in female and male ERAP1+/- and WT mice after 1 week on a restricted-salt diet and liberal-salt diet. Twenty-four-hour urinary ALDO measured by immunoassay on a liberal-salt diet (A and $\mathbf{B})$ or restricted-salt diet (C and $\mathbf{D})$. Data are presented as mean \pm SEM. WT $n=7-10, \mathrm{ERAP}^{+/-} n=9$; 2-tailed Student's $t$ test.

of these mechanisms can lead to increased BP, $\operatorname{SSBP}$, and $\operatorname{HTN}(16,20,21)$. We documented that while BP responses were similar in male and female $\mathrm{ERAP} 1^{+/-}$mice, the likely mechanisms underlying the volume expansion differed by sex.

On a liberal-salt diet, inappropriately elevated ALDO causes retention of sodium and water, leading to blood volume expansion. The normal response to volume expansion is an increase in RPF, leading to an increase in sodium excretion and thus a decrease in volume expansion (17). Countering this response is ANGII, a potent vasoconstrictor and critical regulator of the renal vasculature (17). ANGII can reduce RPF, thus increasing sodium retention on a liberal-salt diet and lead to SSBP.

On a liberal-salt diet, ALDO levels were significantly higher in male ERAP1 ${ }^{+/-}$compared with male WT mice. Male ERAP1 ${ }^{+/-}$mice showed appropriate changes in renovascular function (RI and PI of the left renal artery) to increase sodium excretion and reduce volume. These data suggest that in males, reductions in ERAP1 lead to increased ANGII-stimulated ALDO, which promotes sodium and water retention, leading to SSBP; males showed appropriate compensatory renovascular responses that minimized this effect of ALDO.

Female ERAP1 ${ }^{+/-}$mice, like their male littermates, developed SSBP. But females did not show the same responses as males in terms of ALDO and renovascular function. On a liberal-salt diet, female ERAP1 ${ }^{+/}$ mice did not alter renovascular function (RI and PI of the left renal artery) to increase sodium excretion and reduce volume. Thus, we postulate that SSBP in female ERAP1 ${ }^{+/-}$mice is driven by an ANGII-mediated abnormal renovascular response to a liberal-salt diet. Female ERAP1 ${ }^{+/}$and WT mice displayed similar ALDO levels on a liberal-salt diet, which were significantly higher than levels in males. Of note, we have recently documented that women and female rodents have significantly higher ALDO responses to ANGII than males (16). It may be that WT females, to compensate for these higher ALDO levels, adapt their renovasculature to excrete sodium and water on a liberal-salt diet, thus leading to SSBP. In female ERAP1 ${ }^{+/}$mice, ANGII-mediated effects on the renovasculature prevented this renovascular compensation, leading to SSBP.

To determine how our rodent studies relate to humans, we characterized individuals from the highly environmentally controlled HyperPATH cohort $(16,22,23)$ who were carriers of the ERAP1 rs30187 risk allele. Our results show that as observed in male ERAP1 ${ }^{+/-}$mice, men who carry the rs 30187 risk allele have increased BP on a liberal-salt diet, and this increase follows an allelic dose response. In addition, male risk allele carriers showed an appropriate and compensatory increase in RPF. We observed that rs30187 was associated with reduced ERAP1 mRNA expression in human CV and adrenal tissues in subjects from the GTEx Project Database (24). These results suggest that in men, reduced ERAP1 levels are associated with increased 
A

Female

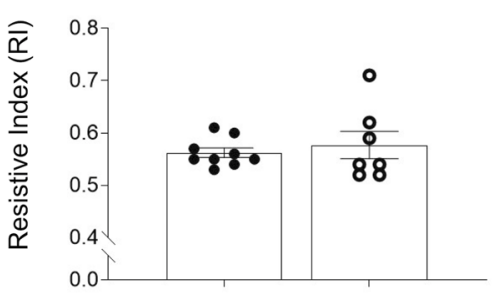

C

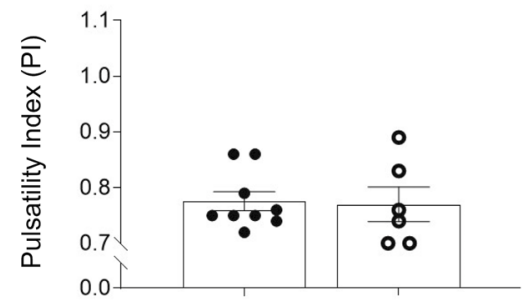

E

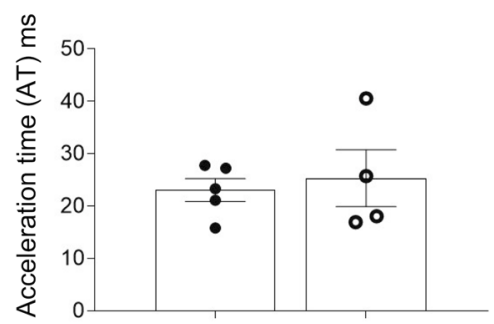

B

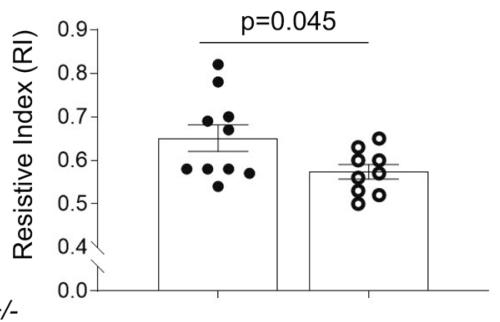

D

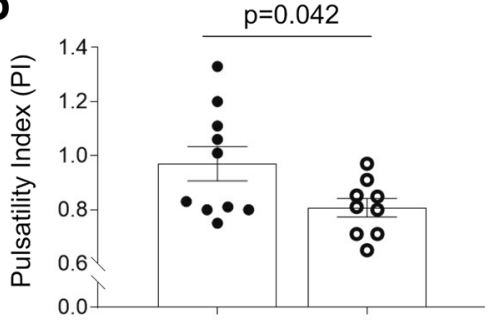

$\mathbf{F}$

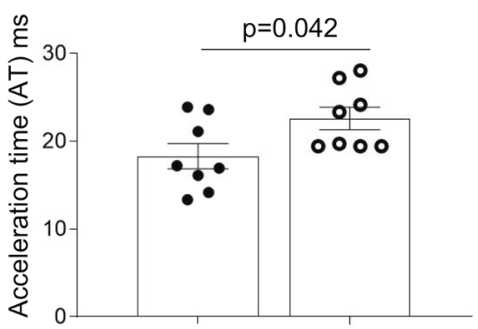

Figure 4. Doppler ultrasound measurements of renovascular function in male and female ERAP1 $^{+/-}$and WT mice on a liberal-salt diet. Renal RI was measured in female (A; WT $n$ $\left.=9, \mathrm{ERAP}^{+/-} n=6\right)$ and male mice (B; $n=10$, $\left.\mathrm{ERAP}^{+/-} n=9, P=0.045\right)$ mice. PI was measured in female (C; WT $n=9$, ERAPP $^{+/-} n=6$ ) and male mice (D;WT $n=10$, ERAP1 ${ }^{+/-} n=9$, $P=0.042)$. Acceleration time was estimated in female $\left(E ;\right.$ WT $n=5$, ERAP1 $\left.{ }^{+-} n=4\right)$ and male mice $\left(\mathbf{F}, \mathrm{WT} n=8\right.$, $\operatorname{ERAP}^{+/-} n=8, P=$ 0.042 ). Data are presented as mean \pm SEM; 2-tailed Student's $t$ test.

$\mathrm{BP}$ and $\mathrm{RPF}$, as observed in male ERAP1 $1^{+/}$mice. Of importance, patients with primary aldosteronism had lower RI and PI as compared with patients with essential HTN, and treatment of primary aldosteronism with adrenalectomy or a mineralocorticoid receptor antagonist led to increases in RI and PI, suggesting that excess ALDO reduces RI and PI (25). These results were not observed in women; female risk allele carriers on a liberal-salt diet did not show changes in BP or RPF as compared with female noncarriers.

Our findings suggest that clinical trials using angiotensin-converting enzyme inhibitors (ACEis) or angiotensin receptor blockers (ARBs) would elicit a better reduction in BP in men than women. However, such differential effects have not been conclusively documented, and thus there is no evidence supporting antihypertensive therapies based on sex $(26,27)$. This is in part due to the fact that there are no studies in which sex differences in ARB or ACEi treatment effectiveness are a primary outcome (28). In fact some, but not all, large clinical trials have reported subgroup analyses of sex-specific outcomes or sex-adjusted analyses, but these results have not been consistent, as detailed in studies involving ARB treatment $(29,30)$. In addition, a recent review cites several genetically mediated subsets of HTN that also may contribute to SSBP and ALDO differences (31). Based on the frequency of the rs30187 risk allele in the HyperPATH cohort, homozygote carriers would be a small fraction of hypertensive subjects that have participated in these aforementioned clinical trials. Thus, if studies are not specifically designed to test for this possibility, the background noise likely would obscure finding such a difference. This issue was also the reason that we excluded homozygote carriers of one of these confounding alleles, $A G T$ rs699, a gene variant likely associated with increased ANGII levels via a different mechanism.

Our study has some potential limitations. First, we and others have documented increased BP and SSBP in aging humans leading to altered RPF and RAS activity (20). Thus, while we controlled for age in our human studies, it may not be sufficient to account for all age effects. Second, our findings on liberal-salt diet may not be relevant to the average diet in humans. However, the liberal salt mouse diet is equivalent to the 200-mM sodium human diet, and the average diet in the United States contains between 130 and $220 \mathrm{mM}$ sodium. Third, we do not know why males and females show different effects. Possible explanations include (i) differences in sex hormone levels; (ii) our inability to determine from the 

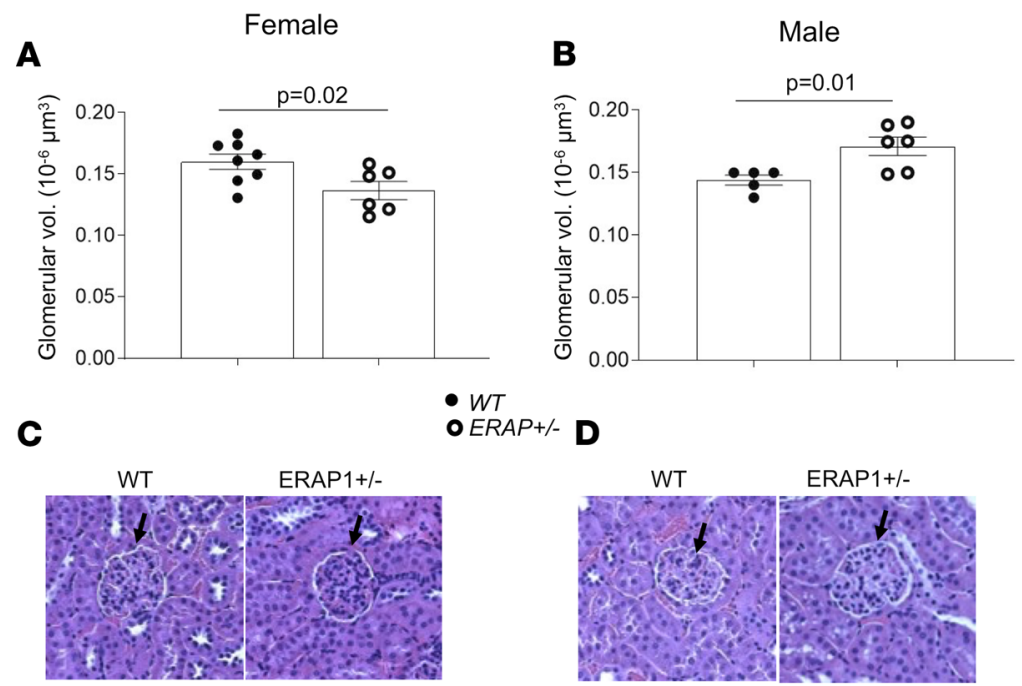

Figure 5. Measurements of glomerular volume in male and female ERAP1 ${ }^{+-}$and $\mathbf{W T}$ mice. Clomerular volume was estimated as described in Methods in female (A; WT $n=8$, ERAP $\left.1^{+-} n=6\right)$ and male mice (B; WT $n=5$, ERAP1 $1^{+/-} n=$ 6). Representative renal histology sections stained with $\mathrm{H} \& \mathrm{E}$ of female (C) and male (D) WT and ERAP1 ${ }^{+/-}$mice; black arrows indicate cross section of glomerulus. Original magnification, $\times 40$. Data are presented as mean \pm SEM; 2 -tailed Student's $t$ test.

HyperPATH database women's menopausal status or menstrual cycle phase, which may have confounding effects; (iii) that ALDO secretagogues other than ANGII could be mediating the differences in ALDO responses to the liberal-salt diet (although the PRA response was equivalent in male and female mice, and PRA and potassium levels were similar in men and women); and (iv) that ERAP2 could play an important and compensating role in women but not men. In fact, ERAP2 gene variants have been shown to be associated with the development of preeclampsia (32). However, mice do not express ERAP2 (33), and the male humans and mice had similar phenotypic characteristics.

In summary, there is growing evidence that biological sex has important effects on CV disease. Our results support the hypothesis that decreasing ERAP1 increases ANGII levels, resulting in increased BP on a liberal-salt diet. However, the underlying mechanisms differ by sex: in males, ALDO is increased; in females, the response of the renal vasculature to volume expansion is impaired. In addition, our results suggest that male rs30187 ERAP1 risk allele carriers, and especially homozygous carriers, may derive more clinical benefit from therapeutic ARB or ACEi use than women. Clinical trials to assess this hypothesis have yet to be performed.

\section{Methods}

Animal model. Ten- to 12-week-old $E R A P 1^{+/-}$mice and littermate $\left(E R A P 1^{+/+}\right)$male mice (B6.129S6-Erap1tm1Luc/J; stock 006413) were purchased from the Jackson Laboratory for breeding. The genotypes were determined by PCR according to the Jackson Laboratory's guidelines and confirmed by Transnetyx. Animals were housed in the animal facility (Brigham and Women's Hospital) on a 12-hour-light/12-hour-dark cycle, at an ambient temperature of $22 \pm 1^{\circ} \mathrm{C}$, and were maintained on Purina Rodent Chow (5053, $0.26 \%$ $\mathrm{Na}^{+}$, Purina) and tap water ad libitum until the beginning of the study.

Sodium intake studies. After 3 days of acclimatization, mice of each genotype from our inbred colony were started on either a liberal salt ( $1.6 \%$ sodium) diet or a restricted salt $(0.03 \%$ sodium) diet for 7 days to achieve sodium balance, as previously reported $(34,35)$.

BP measurement. Systolic and diastolic BP was measured in restrained, conscious mice after reaching a sodium balance on day 5 using tail-cuff plethysmography (CODA High Throughput System, Noninvasive Blood Pressure System, CODA-HT8; Kent Scientific Corp.). Mice were warmed to $30^{\circ} \mathrm{C}$ and allowed to rest quietly. BP measurements were taken in a quiet room, and the mice were kept calm and handled by the same person. No sedation was used. We have previously demonstrated a strong correlation between systolic BPs assessed simultaneously by tail-cuff and telemetry in mice (36). 

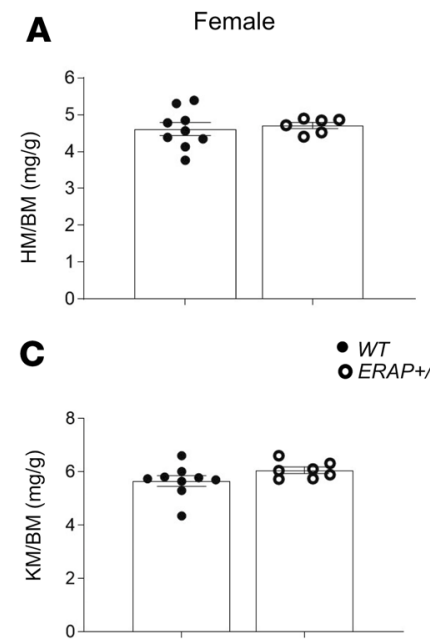

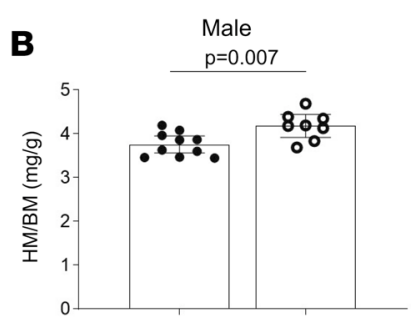

Figure 6. Heart and kidney mass of ERAP1 ${ }^{+/-}$and WT mice relative to body mass. (A) Female heart mass/body mass ratio (HM/BM) (WT $n=9$, ERAP1+/$n=6)$, (B) male HM/BM (WT $n=10$, ERAP1 $\left.^{+/-} n=8\right)$, (C) female kidney mass/ BM ratio (KM/BM) (WT $n=9$, ERAP1 $\left.{ }^{+-} n=7\right)$, and (D) male KM/BM (WT $n=$ 10, $\left.\mathrm{ERAP}^{+/-} n=7\right)$. BM of males: WT $32.1 \pm 0.6 \mathrm{~g}(n=11)$, ERAP1 ${ }^{+/-} 31.2 \pm 0.5$ $\mathrm{g}(n=9)$; females: WT $23.6 \pm 0.4 \mathrm{~g}(n=9)$, ERAP1 ${ }^{+/-} 22.9 \pm 0.5 \mathrm{~g}(n=7)$. Data are presented as mean \pm SEM; 2-tailed Student's $t$ tests.

RT-PCR. RT-PCR was performed as previously described (37). Total mRNA was extracted from hearts and kidneys using the RNeasy Mini Kit (QIAGEN). cDNA was synthesized from 1.5 to $3 \mu \mathrm{g}$ RNA with a First-Strand cDNA Synthesis Kit (GE Healthcare). PCR amplification reactions to detect ERAP1 and housekeeping 18S ribosomal RNA were performed in duplicate using TaqMan gene expression assays (Applied Biosystems) and the QuantStudio 3 Real-Time PCR System (Thermo Fisher Scientific). mRNA expression levels were normalized to $18 \mathrm{~S}$ ribosomal RNA levels, and data are presented as a fold increase relative to measurements in WT mice.

Western blot analysis. Western blot analysis was performed as previously described (38). Tissues were homogenized on ice-cold lysing solution containing RIPA buffer (Boston BioProducts) and $1 \times$ protease inhibitor cocktail (Pierce Biotechnology). ERAP1 primary antibody was purchased from Bioworld (BS2155, diluted 1:2000); secondary antibody used was HRP-conjugated goat anti-rabbit IgG (sc-2004, Santa Cruz Biotechnology Inc., dilution 1:5000). Antibody was detected by ECL (HyGLO Quick Spray Chemiluminescent, Denville Scientific). All Western blots were reprobed with anti- $\beta$-tubulin or anti- $\beta$-actin antibody, and ERAP1 protein levels were normalized to $\beta$-tubulin or $\beta$-actin to correct for loading variability.

Tissue ANGII measurements. Heart, aorta, and kidney tissues were prepared for LC-MS/MS as previously described (39), at the Brigham Research Assay Core. Tissues (100-300 mg) were homogenized in $800 \mu \mathrm{L}$ ice-cold lysis buffer containing $50 \mathrm{mM}$ Tris, $10 \mathrm{mM}$ EDTA, and $1 \times$ protease inhibitor cocktail (Pierce Biotechnology). ANGII in tissue homogenate was extracted by solid phase extraction (SPE), separated and eluted by HPLC, and determined by MS/MS in an electrospray ionization (ESI) source at positive ionization mode with multiple reaction monitoring (MRM) of transition. Stable isotope-labeled ANGII was utilized as an internal standard for assay calibration.

Assays and analyses. Urinary ALDO levels were measured by ELISA kit (IBL International). Urine sodium and potassium were measured by flame photometry per the manufacturer's protocol (Cole-Parmer).

Telemetry probe insertion. A mouse telemetry probe (HD-X11; DSI) was inserted into the aortic arch per the manufacturer's protocol. Starting from 2 weeks after surgery, data were collected following 1 week of a $1.6 \%$ or $0.03 \%$ sodium diet, as previously described in mice (36).

Renal blood flow measurement with noninvasive Doppler ultrasound. Pulse-wave Doppler ultrasound was performed at the Cardiovascular Physiology Core at Brigham and Women's Hospital as previously described (40). Briefly, all animals were acclimatized overnight before experimental procedures were performed. We used inhaled isoflurane ( $2 \%-3 \%$ to induce and $1.0 \%$ to maintain) in this study, which allowed us to obtain consistent and reproducible images and measurements, as previously reported by the Core $(41,42)$. Briefly, renovascular function was assessed by measuring peak systolic $(\mathrm{mm} / \mathrm{s})$ and end diastolic velocities $(\mathrm{mm} / \mathrm{s})$ to estimate RI and PI. The data presented in this study were obtained by a single operator blinded to mouse genotype and interventions at the Core facility. Images were acquired using a Vevo 3100 system (Fujifilm/VisualSonics), and data were analyzed by an investigator blinded to mouse genotype and sex using Vevo LAB software. Mice were studied after 7 days on a liberal-salt diet because the range of RPF responsiveness on liberal salt is greater than on restricted salt, thus facilitating 
Table 1. Characteristics of subjects on liberal-salt diet according to rs30187 genotype

\begin{tabular}{|c|c|c|c|c|}
\hline & Homozygous major allele & Heterozygous & Homozygous minor allele & $P$ value \\
\hline \multicolumn{5}{|l|}{ Men } \\
\hline$n$ & 100 & 116 & 28 & \\
\hline Age (yr) & $43.5 \pm 1.1$ & $45.9 \pm 0.9$ & $45.2 \pm 1.9$ & 0.24 \\
\hline $\mathrm{BMI}(\mathrm{kg} / \mathrm{m})$ & $27.1 \pm 0.4$ & $27.1 \pm 0.4$ & $26.9 \pm 0.7$ & 0.98 \\
\hline HTN (\%) & $74(74.00)$ & $85(73.28)$ & 25 (89.29) & 0.19 \\
\hline ALDO (ng/dL) & $5.4 \pm 0.4$ & $5.5 \pm 0.4$ & $4.9 \pm 0.6$ & 0.81 \\
\hline Serum K+ (mmol/L) & $4.2 \pm 0.04$ & $4.2 \pm 0.04$ & 4.20 .08 & 0.62 \\
\hline Urine $\mathrm{K}^{+}(\mathrm{mmol} / \mathrm{d})$ & $75.3 \pm 3.0$ & $72.9 \pm 2.5$ & 76.13 .6 & 0.75 \\
\hline Urine $\mathrm{Na}^{+}(\mathrm{mmol} / \mathrm{d})$ & $232.8 \pm 8.4$ & $215.2 \pm 7.2$ & $207.8 \pm 9.0$ & 0.15 \\
\hline Urine ALDO ( $\mu \mathrm{g} / 24$ h) & $11.49 \pm 0.88$ & $11.85 \pm 1.32$ & $12.38 \pm 0.84$ & 0.75 \\
\hline \multicolumn{5}{|l|}{ Women } \\
\hline ALDO (ng/dL) & $4.99 \pm 0.41$ & $4.94 \pm 0.40$ & $3.75 \pm 0.54$ & 0.29 \\
\hline ANGII (pg/mL) & $30.0 \pm 2.7$ & $25.0 \pm 2.0$ & $30.5 \pm 4.4$ & 0.32 \\
\hline PRA (ng/mL/h) & $0.47 \pm 0.05$ & $0.61 \pm 0.07$ & $0.41 \pm 0.11$ & 0.15 \\
\hline Serum K+ $(\mathrm{mmol} / \mathrm{L})$ & $4.2 \pm 0.06$ & $4.1 \pm 0.05$ & $4.3 \pm 0.12$ & 0.18 \\
\hline Urine $\mathrm{K}^{+}(\mathrm{mmol} / \mathrm{d})$ & $63.3 \pm 3.0$ & $67.5 \pm 3.3$ & $63.5 \pm 5.6$ & 0.61 \\
\hline Urine $\mathrm{Na}^{+}(\mathrm{mmol} / \mathrm{d})$ & $211.1 \pm 8.6$ & $205.8 \pm 9.0$ & $213.5 \pm 14.6$ & 0.87 \\
\hline Urine ALDO ( $\mu \mathrm{g} / 24 \mathrm{~h})$ & $12.25 \pm 0.94$ & $10.58 \pm 0.75$ & $12.06 \pm 1.43$ & 0.36 \\
\hline
\end{tabular}

Data are presented as mean \pm SEM. One-way ANOVA for continuous variables and $\chi^{2}$ test for categorical variables. Among Black men $(P=0.89)$ and women $(P=0.67)$, respectively: $n=2(2.06 \%)$ and $n=4(5.00 \%)$ were homozygous for the major allele; $n=3(2.65 \%)$ and $n=6(7.79 \%)$ heterozygous; and $n=1$

(3.70\%) and $n=1(3.85 \%)$ homozygous for the minor allele.

detection of potential differences, as reported in humans (17). In addition, our liberal-salt diet closely mimics the average ambient diet in humans and enhances the generalizability of our findings.

Tissue processing and glomerular volume measurement. Tissue section preparation staining was performed at the Histology Core at Harvard Medical School as previously described (43). Briefly, renal tissue from each animal was fixed in buffered $10 \%$ formalin and embedded in paraffin blocks. Kidney sections $(5-\mu \mathrm{m})$ were stained with H\&E and examined by light microscopy by a pathologist blinded to the genotype and treatment type for estimation of glomerular volume, as previously described (43).

Human subjects. Data were evaluated from a cohort of participants of the multisite HyperPATH consortium developed to characterize the genetic underpinnings of HTN. A detailed description of this population has been published previously $(16,23,44-47)$. Data from some of these subjects have been previously reported; however, the analyses and results shown herein have not. HyperPATH was designed to overcome inherent difficulties associated with investigating the genetic basis of complex heritable diseases such as HTN using intermediate, deep phenotyping. This approach achieves homogeneity, thereby amplifying signal/noise ratios, resulting in more robust associations studies with smaller sample sizes; and serves as a foundation for rational candidate gene selection, in this case genes associated with ANGII metabolism such as ERAP1. HyperPATH includes approximately 2000 individuals representing community-dwelling, general hypertensive, and normotensive populations in Boston, Salt Lake City, Nashville, Rome, and Paris.

Screening process. In brief, screening history and physical and laboratory data were obtained from each participant prior to enrollment. Qualified subjects were required to have a personal history of HTN defined as diastolic BP $\geq 100 \mathrm{mmHg}$ on no medications; diastolic BP $\geq 90 \mathrm{mmHg}$ on 1 antihypertensive agent; or the use of 2 or more antihypertensive medications at the time of screening. Subjects on $\geq 4$ antihypertensive medications at the time of screening were excluded. Antihypertensive medications were withdrawn prior to laboratory characterization to reduce their influence on RAS assessment. This included withdrawal for 3 months for converting enzyme inhibitors, angiotensin receptor blockers, and aldosterone antagonists; and at 


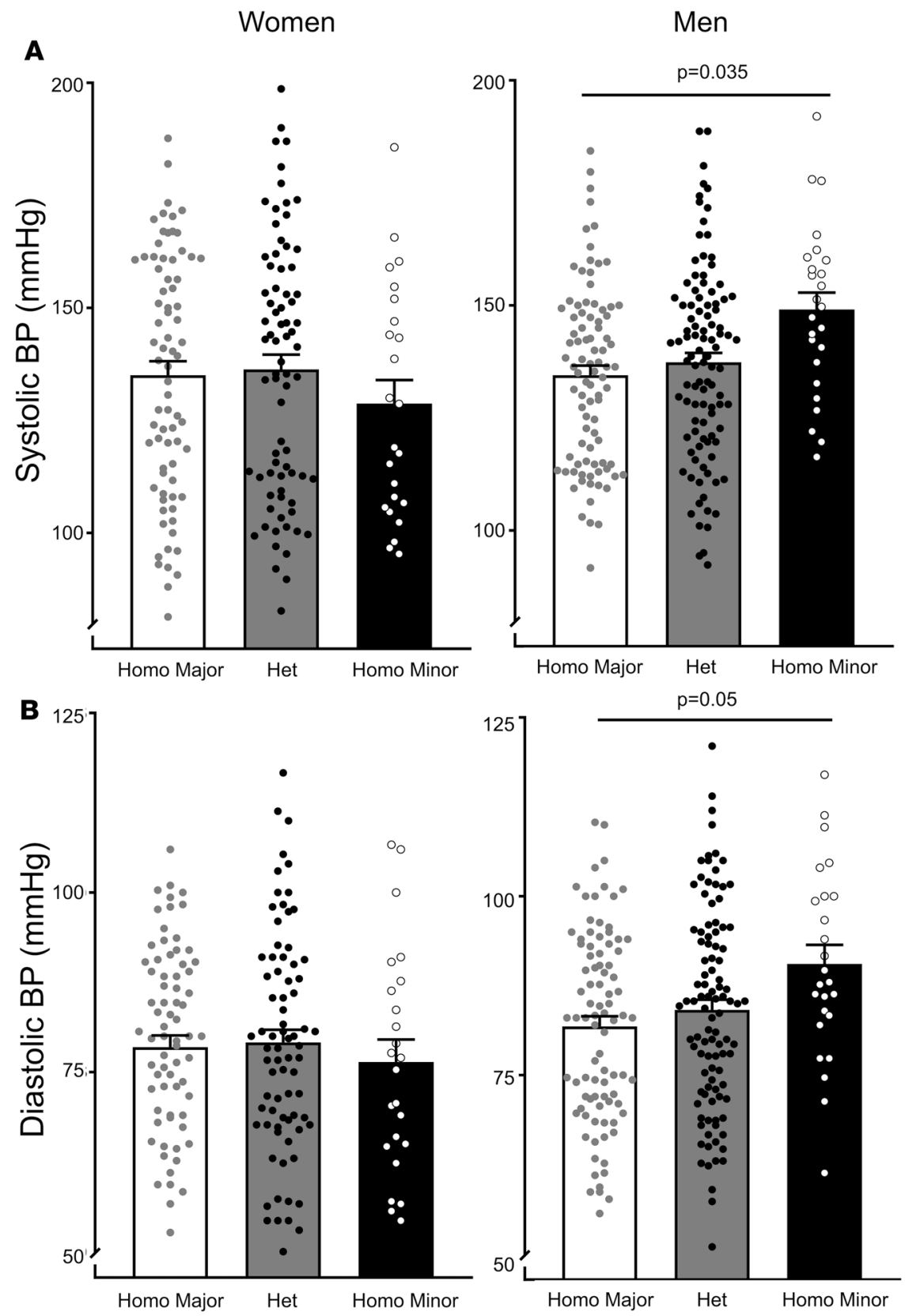

Figure 7. Blood pressures of women and men according to rs30187 genotype. Women and men from the HyperPATH cohort were genotyped according to the rs30187 gene variant as homozygous for the major allele (Homo Major), or heterozygous (Het) or homozygous for the minor allele (Homo Minor), the risk allele. Systolic BP (A) and diastolic BP (B) diastolic BP of females and males. Data are presented as mean $\pm \mathrm{SEM}$. $P$ values were determined from multiple linear regression model analyses adjusted for age, race, BMI, disease state (HTN vs. normal BP), and site (Boston, Salt Lake City, Paris, Rome) for systolic BP coefficient: 3.4, $P=0.035$; and for diastolic BP coefficient: 2.0, $P=0.05$.

least 2 weeks for other antihypertensives. Subjects with secondary HTN assessed at screening, known diabetes mellitus, renal insufficiency, or a significant medical history were excluded from analysis. Subjects studied had normal history and physical, except for HTN, although some $(<10 \%)$ had impaired glucose tolerance. All subjects were between 18 and 65 years old, and race was self-defined. Normotension was defined as seated diastolic $\mathrm{BP} \leq 80 \mathrm{mmHg}$ on 3 different dates at least 1 month apart, with 3 replicates at each date.

Genotyping. DNA was obtained from stored leukocytes, using procedures previously described by us, from the Crimson Biospecimen Core (QIAGEN) (23). Genotyping was done at the Partners HealthCare Center for Personalized Genetic Medicine (PCPGM) Genotyping Facility using the Illumina Bead Station GoldenGate platform. Prior studies documented an association between ERAP1 rs30187 and 


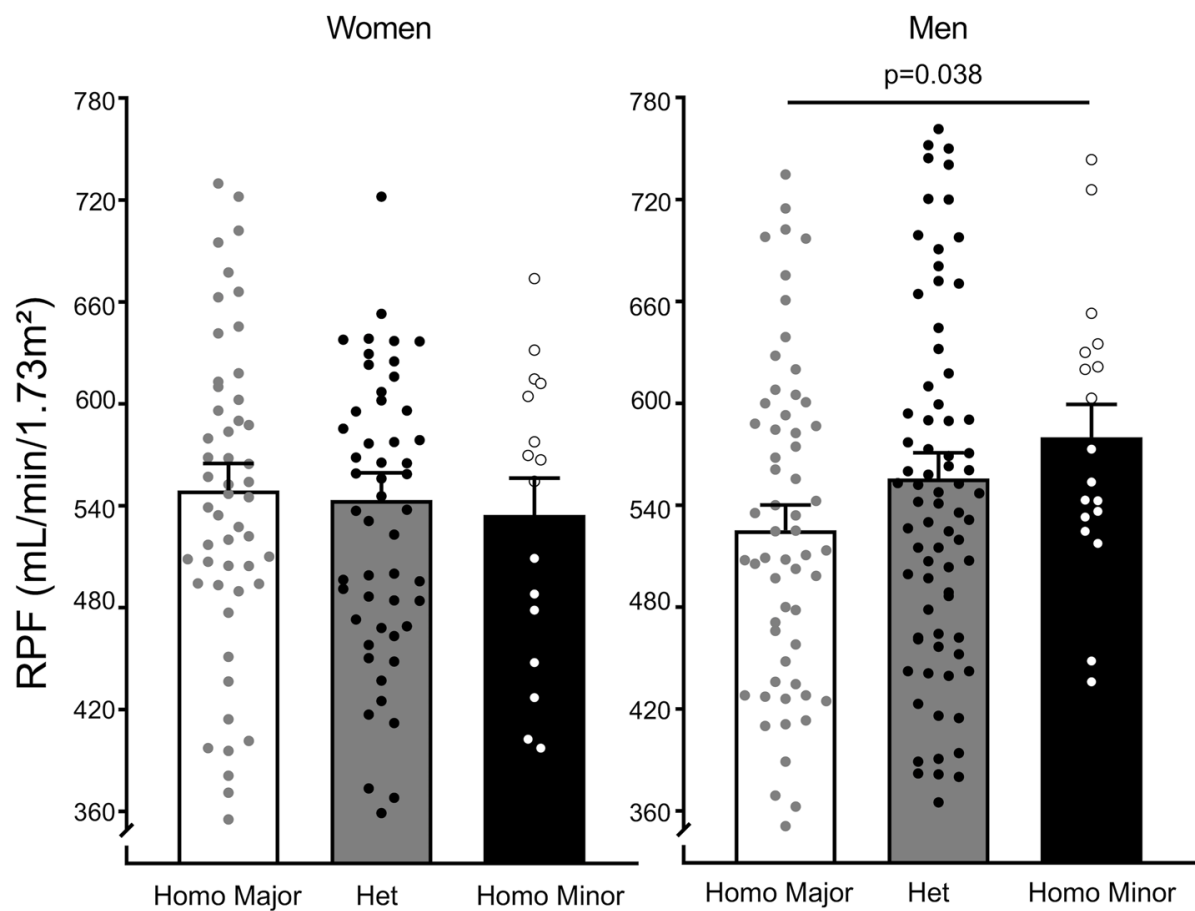

Figure 8. RPF in women and men according to rs30187 genotype. Women and men from the HyperPATH cohort were genotyped according to the rs30187 gene variant as homozygous for the major allele, or heterozygous or homozygous for the minor allele, the risk allele. PAH clearance was used to estimate RPF. Data are presented as mean \pm SEM. $P$ value was determined from multiple linear regression model analyses for RPF in men adjusted for age, race, BMI, disease state (HTN vs. normal BP), and site (Boston, Salt Lake City, Paris, Rome); coefficient: 27, $P=0.038$.

HTN and reduced ERAP1 activity $(5,6,12)$. Therefore, we focused on rs30187 in evaluating the potential effects of the ERAP1 genotype. Participants with a risk allele in this study were defined as carrying the minor allele of rs 30187 . The genotyping success rate was $95 \%$ at this locus. Also, our group and others have documented that subjects homozygous for rs699, a gain-of-function mutation of the angiotensinogen (AGT) gene, have increased levels of AGT, the substrate for renin and thus for ANGII. Therefore, homozygous carriers of the rs699 risk allele were excluded from our analyses (48).

Study protocol. Subjects were provided information and supplements to ensure a daily diet of approximate $200 \mathrm{mM}$ sodium, $100 \mathrm{mM}$ potassium, and 2500 calories for 1 week prior to admission to a General Clinical Research Center (GCRC) for 1 night and 1 day, during which hemodynamic and laboratory assessments occurred between 8 am and 10 am in a fasting, supine state. Laboratory measurements included PRA, ANGII, and ALDO. Blood pressure was recorded by an automatic recording device (Dinamap, Critikon) at 5-minute intervals and averaged for analysis. RPF was determined by PAH clearance as previously reported $(16,23,44-47)$.

Analyte measurements. Hormones and electrolytes in serum and urine were measured in the Brigham and Women's Hospital's Research Assay Core Laboratory as previously reported (22, 23). ALDO levels were measured by using a Coat-A-Count RIA kit (Siemens); PRA by RIA assay (DiaSorin); and ANGII and PAH by LC-MS.

Statistics for mouse studies. Data are expressed as mean \pm SEM. Paired data were compared by 2-tailed Student's $t$ tests. Comparisons between multiple groups were made with 2-way ANOVA followed by Dunnett's post hoc test for multiple comparisons. Data were analyzed using GraphPad Prism 6 software. Differences with $P$ values 0.05 or less were considered statistically significant.

Statistics for human studies. We investigated genotype-phenotype associations in a total of 435 individuals in whom complete data were available. Genotype frequencies were in Hardy-Weinberg equilibrium by $\chi^{2}$ test. Comparisons of frequencies were performed using $\chi^{2}$ tests where appropriate. The study population included Black participants; however, this population was too small to analyze separately. All statistical 
analyses were performed with Stata/SE 15.1 (StataCorp). Data and results are presented as mean \pm SEM and percentages for categorical variables. Univariate analyses were performed using a $\chi^{2}$ test and/or 1 -way ANOVA. We applied a multiple linear regression model to test ERAP1 risk allele associations with the phenotypes (BP and RPF) in those on liberal-salt diets. All association analyses between ERAP1 risk allele and $\mathrm{BP}$ and RPF were performed assuming multiple linear regression model adjusted for age, race, $\mathrm{BMI}$, disease state (HTN vs. normal BP), and sites in subjects from the HyperPATH cohort that had all the appropriate variables. Differences with $P$ values 0.05 or less were considered statistically significant.

Study approval. All experimental animal studies followed the guidelines of, and were approved by, the IACUC at the Brigham and Women's Hospital/Harvard Medical School. All study procedures in humans were approved by the Brigham and Women's Hospital Institutional Review Board, and written informed consent was obtained from participants prior to inclusion in the study.

\section{Author contributions}

SR, JSW, AR, GKA, LHP, GHW, and JRR designed research studies. JWT, KYHW, GHW, and JRR performed human data analyses. SR, JYW, JWT, DLB, AEG, SAM, IAK, CST, JML, AR, LHP, and JRR performed rodent studies and rodent data analyses. SR, JSW, AR, LHP, GHW, GKA, and JRR contributed to data interpretation and manuscript preparation. SR, GKA, GHW, and JRR wrote the manuscript.

\section{Acknowledgments}

We acknowledge financial assistance, in partial support for these studies, from the following sources: Brigham and Women's Hospital, Division of Endocrinology, Diabetes and Hypertension, and Harvard Medical School; NIH grants P50HL055000 (HyperPATH cohort); UL1RR025758, Harvard Clinical and Translational Science Center, from the National Center for Research Resources; M01-RR02635, Brigham and Women's Hospital, General Clinical Research Center, from the National Center for Research Resources; T32 training grant T32HL007609; NIH research grants R01HL086907, R01HL114765, R01HL144779, R01HL136567, R01HL096518, and R01HL104032 from the National Heart, Lung, and Blood Institute; and grants from the National Center for Advanced Translational Science (RM-07-2002) and the American Heart Association (14GRNT20500000).

Address correspondence to: Jose R. Romero, Brigham and Women's Hospital, Division of Endocrinology, Diabetes and Hypertension, 221 Longwood Avenue, Boston, Massachusetts 02115, USA. Phone: 617.732.4948. E-mail: jromero@partners.org.

1. Kurtz TW, DiCarlo SE, Pravenec M, Morris RC. An appraisal of methods recently recommended for testing salt sensitivity of blood pressure. J Am Heart Assoc. 2017;6(4):e005653.

2. Elijovich F, et al. Salt sensitivity of blood pressure: a scientific statement from the American Heart Association. Hypertension. 2016;68(3):e7-e46.

3. Mente A, et al. Associations of urinary sodium excretion with cardiovascular events in individuals with and without hypertension: a pooled analysis of data from four studies. Lancet. 2016;388(10043):465-475.

4. O'Donnell M, et al. Dietary sodium and cardiovascular disease risk. N Engl J Med. 2016;375(24):2404-2406.

5. Goto Y, Hattori A, Ishii Y, Tsujimoto M. Reduced activity of the hypertension-associated Lys528Arg mutant of human adipocyte-derived leucine aminopeptidase (A-LAP)/ER-aminopeptidase-1. FEBS Lett. 2006;580(7):1833-1838.

6. Tsujimoto M, Goto Y, Maruyama M, Hattori A. Biochemical and enzymatic properties of the M1 family of aminopeptidases involved in the regulation of blood pressure. Heart Fail Rev. 2008;13(3):285-291.

7. Hattori A, et al. Characterization of recombinant human adipocyte-derived leucine aminopeptidase expressed in Chinese hamster ovary cells. J Biochem. 2000;128(5):755-762.

8. Cui X, et al. Identification of ARTS-1 as a novel TNFR1-binding protein that promotes TNFR1 ectodomain shedding. J Clin Invest. 2002;110(4):515-526.

9. Cifaldi L, et al. ERAP1 regulates natural killer cell function by controlling the engagement of inhibitory receptors. Cancer Res. 2015;75(5):824-834

10. Goto Y, Ogawa K, Hattori A, Tsujimoto M. Secretion of endoplasmic reticulum aminopeptidase 1 is involved in the activation of macrophages induced by lipopolysaccharide and interferon-gamma. J Biol Chem. 2011;286(24):21906-21914.

11. López de Castro JA, Alvarez-Navarro C, Brito A, Guasp P, Martín-Esteban A, Sanz-Bravo A. Molecular and pathogenic effects of endoplasmic reticulum aminopeptidases ERAP1 and ERAP2 in MHC-I-associated inflammatory disorders: towards a unifying view. Mol Immunol. 2016;77:193-204.

12. Yamamoto N, Nakayama J, Yamakawa-Kobayashi K, Hamaguchi H, Miyazaki R, Arinami T. Identification of 33 polymorphisms in the adipocyte-derived leucine aminopeptidase (ALAP) gene and possible association with hypertension. Hum Mutat $2002 ; 19(3): 251-257$ 
13. Hisatsune C, et al. ERp44 exerts redox-dependent control of blood pressure at the ER. Mol Cell. 2015;58(6):1015-1027. 14. Vaidya A, et al. Abnormal aldosterone physiology and cardiometabolic risk factors. Hypertension. 2013;61(4):886-893.

15. Baudrand R, et al. Caveolin 1 modulates aldosterone-mediated pathways of glucose and lipid homeostasis. J Am Heart Assoc. 2016;5(10):e003845

16. Shukri MZ, et al. Biological sex modulates the adrenal and blood pressure responses to angiotensin II. Hypertension. 2018;71(6):1083-1090.

17. Hollenberg NK, Moore T, Shoback D, Redgrave J, Rabinowe S, Williams GH. Abnormal renal sodium handling in essential hypertension. Relation to failure of renal and adrenal modulation of responses to angiotensin II. Am J Med. 1986;81(3):412-418.

18. Petersen LJ, Petersen JR, Talleruphuus U, Ladefoged SD, Mehlsen J, Jensen HA. The pulsatility index and the resistive index in renal arteries. Associations with long-term progression in chronic renal failure. Nephrol Dial Transplant. 1997;12(7):1376-1380.

19. Carithers LJ, Moore HM. The Genotype-Tissue Expression (GTEx) project. Biopreserv Biobank. 2015;13(5):307-308.

20. Brown JM, et al. Aldosterone dysregulation with aging predicts renal vascular function and cardiovascular risk. Hypertension 2014;63(6):1205-1211.

21. Whaley-Connell AT, et al. Salt loading exacerbates diastolic dysfunction and cardiac remodeling in young female Ren2 rats. Metab Clin Exp. 2013;62(12):1761-1771.

22. Gupta T, et al. Striatin gene polymorphic variants are associated with salt sensitive blood pressure in normotensives hypertensives. Am J Hypertens. 2017;31(1):124-131.

23. Tan JW, et al. Dysregulated aldosterone secretion in persons of African descent with endothelin-1 gene variants. JCI Insight. 2017;2(23):95992.

24. GTEx Consortium. Human genomics. The Genotype-Tissue Expression (GTEx) pilot analysis: multitissue gene regulation in humans. Science. 2015;348(6235):648-660.

25. Sechi LA, Di Fabio A, Bazzocchi M, Uzzau A, Catena C. Intrarenal hemodynamics in primary aldosteronism before and after treatment. J Clin Endocrinol Metab. 2009;94(4):1191-1197.

26. Mills KT, et al. Global disparities of hypertension prevalence and control: a systematic analysis of population-based studies from 90 countries. Circulation. 2016;134(6):441-450.

27. Turnbull F, et al. Do men and women respond differently to blood pressure-lowering treatment? Results of prospectively designed overviews of randomized trials. Eur Heart J. 2008;29(21):2669-2680.

28. Rabi DM, Khan N, Vallee M, Hladunewich MA, Tobe SW, Pilote L. Reporting on sex-based analysis in clinical trials of angiotensin-converting enzyme inhibitor and angiotensin receptor blocker efficacy. Can J Cardiol. 2008;24(6):491-496.

29. Trenkwalder P, et al. The Study on COgnition and Prognosis in the Elderly (SCOPE) - major CV events and stroke in subgroups of patients. Blood Press. 2005;14(1):31-37.

30. Zanchetti A, et al. Outcomes in subgroups of hypertensive patients treated with regimens based on valsartan and amlodipine: an analysis of findings from the VALUE trial. J Hypertens. 2006;24(11):2163-2168.

31. Manosroi W, Williams GH. Genetics of human primary hypertension: focus on hormonal mechanisms. Endocr Rev. 2019;40(3):825-856

32. Johnson MP, et al. The ERAP2 gene is associated with preeclampsia in Australian and Norwegian populations. Hum Genet. 2009;126(5):655-666.

33. Saveanu L, et al. Concerted peptide trimming by human ERAP1 and ERAP2 aminopeptidase complexes in the endoplasmic reticulum. Nat Immunol. 2005;6(7):689-697.

34. Hall JE. Control of sodium excretion by angiotensin II: intrarenal mechanisms and blood pressure regulation. Am J Physiol. 1986;250(6 pt 2):R960-R972.

35. Oliverio MI, Best CF, Smithies O, Coffman TM. Regulation of sodium balance and blood pressure by the AT(1A) receptor for angiotensin II. Hypertension. 2000;35(2):550-554.

36. Pojoga LH, et al. Caveolin-1 ablation reduces the adverse cardiovascular effects of N-omega-nitro-L-arginine methyl ester and angiotensin II. Endocrinology. 2010;151(3):1236-1246.

37. Pojoga LH, et al. Effect of dietary sodium on vasoconstriction and eNOS-mediated vascular relaxation in caveolin-1-deficient mice. Am J Physiol Heart Circ Physiol. 2008;294(3):H1258-H1265.

38. Chong C, et al. Regulation of aldosterone secretion by mineralocorticoid receptor-mediated signaling. J Endocrinol. 2017;232(3):525-534.

39. Ali Q, Wu Y, Nag S, Hussain T. Estimation of angiotensin peptides in biological samples by LC/MS method. Anal Methods. 2014;6(1):215-222.

40. Fisch S, Liao R, Hsiao LL, Lu T. Early detection of drug-induced renal hemodynamic dysfunction using sonographic technology in rats. $J$ Vis Exp. 2016(109):e52409.

41. Bjornerheim R, Grogaard HK, Kjekshus H, Attramadal H, Smiseth OA. High frame rate Doppler echocardiography in the rat: an evaluation of the method. Eur J Echocardiogr. 2001;2(2):78-87.

42. Zhang L, et al. A high-frequency, high frame rate duplex ultrasound linear array imaging system for small animal imaging IEEE Trans Ultrason Ferroelectr Freq Control. 2010;57(7):1548-1557.

43. Guo C, et al. Mineralocorticoid receptor antagonist reduces renal injury in rodent models of types 1 and 2 diabetes mellitus Endocrinology. 2006;147(11):5363-5373.

44. Hopkins PN, et al. Blunted renal vascular response to angiotensin II is associated with a common variant of the angiotensinogen gene and obesity. J Hypertens. 1996;14(2):199-207.

45. Hopkins PN, et al. Angiotensinogen genotype affects renal and adrenal responses to angiotensin II in essential hypertension. Circulation. 2002;105(16):1921-1927.

46. Giacché M, et al. Aldosterone stimulation by angiotensin II : influence of gender, plasma renin, and familial resemblance. Hypertension. 2000;35(3):710-716.

47. Vaidya A, et al. The influence of body mass index and renin-angiotensin-aldosterone system activity on the relationship between 25-hydroxyvitamin D and adiponectin in Caucasian men. Eur J Endocrinol. 2011;164(6):995-1002.

48. Jeunemaitre X, et al. Molecular basis of human hypertension: role of angiotensinogen. Cell. 1992;71(1):169-180. 